\title{
Microsymposia
}

Comparison of surface fractal features of thermophilic and mesophilic proteins

Suryanarayanarao Ramakumar, ${ }^{\mathrm{a}, \mathrm{b}}$ Pranjal Mahanta, ${ }^{a}$ Siddhartha Gupta, ${ }^{\mathrm{a}}$ Manoj Bhat $\mathrm{K},{ }^{\mathrm{a}}{ }^{\mathrm{a}}$ Department of Physics, Indian Institute of Science, Bangalore-560012, (India). ${ }^{\mathrm{b}}$ Bioinformatics Centre, Indian Institute of Science, Bangalore-560012, (India). Email: ramak@ physics.iisc.ernet.in

Understanding the adaptation of proteins to high temperature continues to be a subject of intense study. Several factors have been proposed as contributing to protein thermo stability and it is of interest to understand the interplay of the factors causing stability at high temperature. Surface texture is an important structural feature since it plays roles in protein stability, diffusion, protein self-association, recognition of different subunits, macromolecules and ligands. Different types of structural and geometric patterns are present on the protein surface that are implicated in interactions with other proteins or nucleic acids or ligands or small molecules. Mutations on the surface of a given protein have been used to increase the thermal stability of the protein. Also, interactions forming on the outer surface of a protein are likely to be preferred during the course of evolution. Hence, the quantification of protein surface may yield valuable insights concerning stability and functionality of proteins. However, despite protein surface being important, a quantitative evaluation of surface smoothness of (hyper)thermophilic and mesophilic proteins has not been reported so far prompting us to carry out a comparative investigation of the surface features of different classes of proteins using the concept of fractal geometry. The 3D structures for all the proteins, selected for the analysis, had been determined using X-ray crystallographic methods and were retrieved from the protein data bank (PDB). Structures determined using NMR methods and modeled structures were excluded from the data sets. The dataset was additionally filtered such that upon superposition of corresponding $\mathrm{C}_{\alpha}$ atoms, the root mean square deviation (RMSD) between the (hyper)thermophilic and their homologous mesophilic proteins is not more than $5 \AA$ and chain lengths differ by not more than 30 amino acid residues. Here we compared the extent of corrugation or surface smoothness of proteins from (hyper)thermophilic and mesophilic organisms, quantified in terms of surface fractal dimension. The analysis clearly revealed that, moderate thermophilic proteins have significantly lower surface fractal dimension or greater surface smoothness (2.241 versus 2.261, $\left.p=3.6 \times 10^{-4}\right)$ than their counterparts from mesophilic organisms in a two tail paired distribution at the 0.05 level. Importantly and unlike several other structural metrics, surface smoothness enables a clean discrimination between proteins from moderate thermophiles and their homologous proteins from mesophilic organisms. Interestingly, for hyperthermophiles, surface smoothness is not significantly different from that of their mesophilic counterparts perhaps suggesting that the strategies adopted by hyperthemophilic proteins to achieve thermostability may be different from those adopted by proteins from moderately thermophilic organisms. This could be a direct consequence of the temperature dependence of various interactions which stabilize proteins. Our analysis strongly suggest that moderate thermophiles and hyperthermophiles should be treated separately in any comparative study concerning thermostability of proteins. The results obtained here could be exploited for the engineering of proteins with increased thermostability for practical applications.

Keywords: fractal, thermophile, mesophile

\section{Controlling stress in GaN-on-Si}

Alois Krost Otto-von-Guericke-Universität Magdeburg, Institut für Experimentelle Physik, Universitätsplatz 2, D-39106 Magdeburg (Germany). E-mail: alois.krost@ovgu.de

GaN-on-Si heteroepitaxy has become a serious alternative to established growth of group-III-nitrides on sapphire or SiC because of its low price, large diameter wafers, and thermally well conducting properties. Most of the work and published device results have been for GaN growth on (111) silicon substrates. Recently, we have demonstrated thick, crack-free layers exceeding $14 \mu \mathrm{m}$ in thickness. Meanwhile, big companies as Samsung1 and Bridgelux2 have announced breakthroughs in GaN-on-Si-based high-power LEDs aimed for general lighting. For such application thick, high-quality structures with highly conducting n-type layers are required which is a major challenge because of tensile thermal stress leading to cracks in the $\mathrm{GaN} / \mathrm{Si}$ system during cooling down. In addition to thermal stress doping with $\mathrm{Si}$ is a major source of tensile stress. The latter can be overcome by Ge-doping instead of Si-doping3. We show ways to control stresses and strains in $\mathrm{GaN}$ heteroepitaxy to achieve crack-free, device-relevant GaN layers on Si with thicknesses up to $>14 \mu \mathrm{m}$. New fields are growth of semipolar GaN on high-index $\mathrm{Si}(\mathrm{h} 11)$ substrates as well as the realization of GaN/AllnN based microcavities with InGaN/GaN quantum wells for optoelectronic devices such as a blue VCSELs or a polariton lasers turned in worldwide focus of interest. We have grown first mirror structures on Si substrates. However, the growth of AlInN/GaN DBR layers with perfect interfaces turned out to be much more difficult than expected. With small deviations from the lattice-matching conditions in the order of $1 \%$ after a certain thickness of around $30 \mathrm{~nm}$ the upper AlInN interfaces become very rough similar to a Stranski-Krastanov transition whereas the lower ones remain flat. The roughness is smoothened by the subsequent GaN layers. This effect occurs in both compressively or tensely strained conditions on sapphire as well as on silicon substrates.

[1] Tak et. al, Contribution A3.6, IWN 2010, Tampa, Florida (USA). [2] http:// bridgelux.com/media-center/press-releases/bridgelux_demonstrates-dramaticadvancements-in-gan-on-silicon-technology-for-solid-state-lighting/ 2011. [3] A. Dadgar, J. Bläsing, A. Diez, A. Krost, Appl. Phys. Express 2011, 4, 011001. [4] A. Krost, C. Berger, P. Moser, J. Bläsing, A. Dadgar, C. Hums, T. Hempel, B. Bastek, P. Veit, J. Christen, Semiconductor Science and Technology 2011, 26, 014041.

\section{Keywords: GaN on Silicon, stress control, heteroepitaxy}

\section{MS.28.2}

Acta Cryst. (2011) A67, C73-C74

Diffuse x-ray scattering from defects in GaN epitaxial layers Václav Holý, Mykhailo Barchuk, Department of Condensed Matter Physics, Charles University in Prague, Prage (Czech Republic). Email: holy@mag.mff.cuni.cz

GaN epitaxial layers are widely investigated nowadays because of their broad application in electronics and optoelectronics. The limiting parameters of the optoelectronic performance of these materials are their structural quality and densities of various defect types, so that a reliable non-destructive method for the quality assessment of these layers is of a great importance.

In c-oriented wurtzite $\mathrm{GaN}$ layers, screw and edge dislocations perpendicular to the surface (threading dislocations) represent the most 


\section{Microsymposia}

common defect type, while in the non-polar a-GaN layers most of the defects are (0001)-oriented stacking faults [1].

We developed a method for the determination of the densities of individual defect types both in c- and a-oriented GaN layers, based on diffuse x-ray scattering and reciprocal space mapping. In the c-GaN layers the method is based on the comparison of the reciprocal space map of the diffusely scattered intensity measured both in standard and grazing-incidence geometry, with simulations based on a numerical Monte-Carlo procedure [2], [3]. The density and type of stacking faults in a-GaN layers can be determined from the intensity distribution along [0001]-oriented streaks in reciprocal space perpendicular to the fault planes.

We have measured a series of $\mathrm{GaN}$ layers of both orientations with various defect densities and compared the densities resulting from our procedure with results of other independent methods (transmission electron micrography, etching techniques) and we obtain comparable total densities of defects.

[1] M.A. Moram, M.E. Vickers, Reports on Progress in Physics 2009, 72, 036502. [2] M. Barchuk, V. Holý, B. Miljevic, B. Krause, T. Baumbach, J. Hertkorn, F. Scholz, J. Appl. Phys. 2010, 108, 043521. [3] M. Barchuk, V. Holý, B. Miljevic, B. Krause, T. Baumbach, Appl. Phys. Lett. 2011, 98, 021912.

Keywords: gallium nitride, diffuse X-ray scattering, defects

\section{MS.28.3}

Acta Cryst. (2011) A67, C74

Lithium niobate: a smart material for various applications in optoelectronics

Marc D Fontana, Sabrina Mignoni, Rachid Hammoum, Patrice Bourson, Laboratoire Matériaux Optiques, Photonique et Systèmes, Université Paul Verlaine - Metz and Supélec, 2 rue Edouard Belin, 57070 Metz (France). E-mail: fontana@metz.supelec.fr

$\mathrm{LiNbO}_{3}(\mathrm{LN})$ is a widely studied optoelectronic material useful for a great variety of applications in non linear optics, integrated optics and solid state lasers [1], [2]. It can be grown in large single crystals, usually by Czochralski technique, and generally in the congruent composition [3]. Therefore it presents a Li deficiency and thus can be easily doped with various impurities. Doping these crystals can suitably change their optical properties [2]. Thus doping LN with rare earth ions or $\mathrm{Cr}$ is appropriate to induce luminescence. The introduction of $\mathrm{Fe}$ gives rise to a large photorefractive effect, whereas doping with $\mathrm{Mg}$ or $\mathrm{Zn}$ provides electrooptic coefficients with an efficient use [1], [2].

In fact the incorporation of dopants induces a change in the local structure of intrinsic (lattice) defects related to non-stoichiometry [4]. The congruent composition corresponds to a composition of nearly 0.94 , meaning that about $6 \%$ of $\mathrm{Li}$ sites are empty. Since $\mathrm{Nb}^{5+}$ ions have a radius close to $\mathrm{Li}^{+}$, they can go fill these empty sites (they are called antisites defects). Different models were proposed to describe the intrinsic defects in the nominally pure crystals with different compensation mechanisms: Li vacancy model or $\mathrm{Nb}$ vacancy model for the congruent composition [3] and coexisting $\mathrm{Li}$ and $\mathrm{Nb}$ vacancies with a content varying with the composition from congruent to stoichiometric [5]. The incorporation of dopants in the LN lattice does not have the same effect since it enters the A site (generally occupied by $\mathrm{Li}$ ions) or the $\mathrm{B}$ site (generally occupied by $\mathrm{Nb}$ ions) according to the nature and the concentration of the dopants. As a consequence, it induces different changes in the dynamics of intrinsic defects: $\mathrm{Nb}$ and Li vacancies, $\mathrm{Nb}$ antisites.

Here are pointed out the dependencies of several linear and non linear optical properties on the dopant content. The electrooptic and photorefractive [6-8] properties recorded in LN crystals doped with $\mathrm{Mg}, \mathrm{Fe}, \mathrm{Zn}$ or Hf [9], [10] are especially reported and discussed. The link between the mechanism and site of incorporation of dopants and the non-monotonous behaviour of optical properties is demonstrated. Thus the remove of antisite defects is related to a threshold in the behaviour of some optical properties.

At last is shown how the optimization of the structure can be used in applications in optoelectronics.

[1] E. Kratzig, O.F. Schirmer, Photorefractive Materials and Their Applications, Topics in AppliedPhysics, Springer-Verlag, Berlin, 1999, 62, 131-166. [2] T. Volk, M. Wöhlecke, Lithium Niobate Defects, Photorefraction and Ferroelectric Switching, Springer Berlin Heidelberg, 2008. [3] A. Räuber, Chemistry and physics of lithium niobate, Current Topics in Material Sciences, Ed. by E. Kaldis, North Holland, Amsterdam, 1978, 481-601. [4] Y. Zhang, L. Guilbert, P. Bourson, K. Polgar, M.D. Fontana, J. Phys.: Condens. Matter 2006, 18, $957-$ 963. [5] F. Abdi, M.D. Fontana, M. Aillerie, P. Bourson, Applied Physics A 2006, 83, 3, 427-434. [6] M. Abarkan, M. Aillerie, J.-P. Salvestrini, M.D. Fontana, E. Kokanyan, Applied Physics B: Lasers and Optics 2008, 92, 603-608. [7] R. Mouras, M.D. Fontana, M. Mostefa, P. Bourson, J. Opt. Soc. Am. B 2006, 23, 3, 1867-1871. [8] F. Abdi, M. Aillerie, P. Bourson, M.D. Fontana, Journal of Applied Physics, 2009, 106, 033519. [9] R. Hammoum, M.D. Fontana, M. Gilliot, P. Bourson, E. Kokanyan, Solid State Communications, 2009, 149, 1967-1970. [10] S. Mignoni, M.D. Fontana, M. Bazzan, M.V. Ciampolillo, A.M. Zaltron, N. Argiolas, C. Sada, Applied Physics B: Lasers and Optics, 2010, 101, 541-546.

Keywords: defect, optoelectronics, doped

MS.28.4

Acta Cryst. (2011) A67, C74-C75

Study of the thermal anisotropy of $\mathrm{KLu}\left(\mathrm{WO}_{4}\right)_{2}$ for high power laser applications

M. Aguiló, M. C. Pujol, X. Mateos, J. J. Carvajal, F. Díaz, Física i Cristal-lografia de Materials $i$ Nanomaterials (FiCMA-FiCNAEMAS), Universitat Rovira i Virgili (URV), Campus Sescelades, c/ Marcel.li Domingo, s/n, E-43007 Tarragona, (Spain). E-mail: Magdalena.aguilo@urv.cat

$\mathrm{KLu}\left(\mathrm{WO}_{4}\right)_{2}$ crystal belongs to the monoclinic system, with the space group $C 2 / c$. This material is a well known interesting laser host material for optically active lanthanides ions, such as $\mathrm{Yb}^{3+}$ and $\mathrm{Tm}^{3+}$. Successful laser action of both ions, at 1.1 microns and 1.9 microns has been already demonstrated, in different configurations such as slab, waveguide and thin disk and also, in continuous-wave (cw) and pulsed regimes. Nevertheless, the complete knowledge of the physical anisotropy of this material and its application in laser action can lead to further improvements in laser efficiency or new applications. In relation with this aspect, the study of the thermal anisotropy of this material is crucial. Thermal conductivity in this crystal is a symmetrical second-rank tensor and as the crystal is monoclinic it has four nonzero components in the crystallo-physical frame $X_{1 \kappa} / / a$., $X_{2 \kappa} / / \boldsymbol{b}, X_{3 \kappa} / / \boldsymbol{c}^{*}$. With the measurement of the thermal diffusivity along four crystallographic directions $\left(\boldsymbol{a}, \boldsymbol{b}, \boldsymbol{c}\right.$ and $\left.\boldsymbol{c}^{*}\right)$, and the specific heat capacity, the thermal conductivity tensor has been calculated.

$\mathrm{KLuW}$ is grown from high temperature solutions. The solvent used by us is $\mathrm{K}_{2} \mathrm{~W}_{2} \mathrm{O}_{7}$ and the growth method was the Top Seeded Solution Growth by Slow Cooling (TSSG-SC). Samples for each measurement are crystallographically oriented to be cut and polished to optical grade quality. The eigenvalues of the thermal conductivity are $\kappa{ }_{11}=2.95 \mathrm{Wm}^{-}$ ${ }^{1} \mathrm{~K}^{-1}, \kappa{ }_{22}=2.36 \mathrm{Wm}^{-1} \mathrm{~K}^{-1}$, and $\kappa{ }_{33}=4.06 \mathrm{Wm}^{-1} \mathrm{~K}^{-1}$, with the maximum value along a direction in the $\boldsymbol{a}-\boldsymbol{c}$ crystallographic plane, at $40.75^{\circ}$ from the $N_{g}$ principal optical axis ( $22.25^{\circ}$ from c crystallographic axis), figure 1 shows the location of the tensor. KLuW is a optical biaxial crystal, in which the reference system for laser applications and linear optics is the real refractive index part, so the three principal optical directions, 